\title{
Characterization of Metal Doped Polymer Capsules using Confocal Micro X-ray Fluorescence Spectroscopy and X-ray Computed Tomography
}

\author{
Nikolaus L. Cordes ${ }^{1}$, George J. Havrilla ${ }^{2}$, Kimberly A. Obrey ${ }^{1}$, Brian M. Patterson ${ }^{1}$ \\ 1. Los Alamos National Laboratory, Materials Science and Technology Division, Los Alamos, NM USA \\ ${ }^{2}$. Los Alamos National Laboratory, Chemistry Division, Los Alamos, NM USA
}

Defect Induced Mix Experiment (DIME) spherical capsules, utilized as National Ignition Facility (NIF) targets, are composed of a $42 \mu \mathrm{m}$-thick polymer shell which has been doped with a $2 \mu \mathrm{m}$-thick inner layer of 1.5 at.\% germanium and separate $2 \mu \mathrm{m}$-thick outer layer of 1.5 at.\% gallium. The metal-doped layers are separated by a $3 \mu \mathrm{m}$-thick polymer layer. The LANL DIME campaign requires that the characterization of these capsules must provide better accuracy than the fabrication tolerances. X-ray characterization techniques, such as confocal micro X-ray fluorescence (MXRF) spectroscopy and micro- and nano-scale computed tomography (CT) allow for the nondestructive elemental and spatial characterization, respectively, of such samples.

Confocal MXRF was performed using a custom-built confocal MXRF instrument [1-3] with a spatial resolution of $30 \times 30 \times 50 \mu \mathrm{m}$. Line scans of DIME capsules were performed in the y direction at $3 \mu \mathrm{m}$ intervals over a $200 \mu \mathrm{m}$ distance. The capsule was then rotated one degree and the y-direction line scan repeated; this procedure was repeated over two full sample rotations $\left(720^{\circ}\right)$ [4]. This procedure was used to keep instrument artifacts to a minimum. The counts of the $y$-direction line scan were then summed and averaged as a function of sample rotation (see Fig. 2). Micro-CT was performed using a Micro XCT instrument (Xradia, Pleasanton, CA); the voxel sizes of reconstructed micro-CT slices are $600 \mathrm{~nm}$, however the dopant layers were not completely distinct. Nano-CT was performed using an Ultra XRM (Xradia, Pleasanton, CA); the voxel sizes of reconstructed nano-CT slices are $65 \mathrm{~nm}$, which was more than enough resolution to resolve the layers and directly measure the dopant layer thickness.

Figure 1 shows an X-ray fluorescence spectrum using confocal MXRF of a DIME capsule, acquired at a $1.3 \mathrm{~mm}$ distance from the capsule outer edge. The $\mathrm{K}_{\alpha}$ emission lines of both $\mathrm{Ga}$ and Ge are detected. The averaged summation of $\mathrm{Ga} \mathrm{K}_{\alpha}$ and $\mathrm{Ge} \mathrm{K}_{\alpha}$ emission counts, as a function of sample rotation, (Fig. 2), indicates small variations of $\mathrm{Ga}$ and Ge concentrations within the DIME capsule. Preliminary statistical analyses of confocal MXRF data (not shown) acquired from four separate DIME capsules indicate no significant sample-to-sample variations in Ga and Ge concentrations. Reconstructed micro- scale and nano- scale CT slice images (Fig. 3) resolve the metal doped and polymer regions, allowing for the nondestructive quantification of the metal doped and polymer region thicknesses. Preliminary analysis of nano-CT data reveal a $44 \mu \mathrm{m}$-thick polymer shell, and Ga- and Ge-doped region thicknesses of 1.5 and $2.4 \mu \mathrm{m}$. The combination of these three characterization techniques allows for the elemental and spatial resolution of DIME capsules required by the LANL DIME campaign.

\section{References:}

[1] BM Patterson, J Campbell, GJ Havrilla (2010) X-Ray Spectrometry 39: 184.

[2] BM Patterson, GJ Havrilla, KA DeFriend (2008) Microscopy Today 16: 38.

[3] BM Patterson, KAD Obrey, CE Hamilton, GJ Havrilla (2012) X-Ray Spectrometry 41: 253.

[4] BM Patterson, KA Obrey, GJ Havrilla (2011) Fusion Sci. and Technol. 59: 121. 


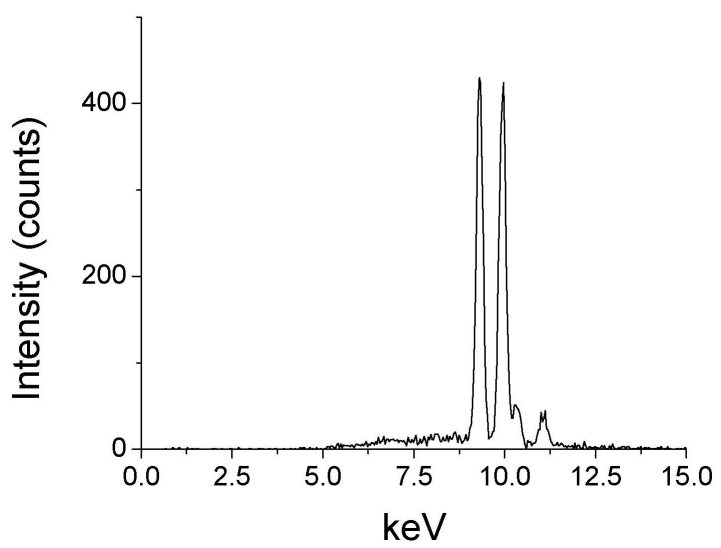

Figure 1. Confocal MXRF spectrum of a DIME capsule acquired at $1.3 \mathrm{~mm}$ from the capsule outer edge. Both $\mathrm{Ga}(9.30 \mathrm{keV})$ and $\mathrm{Ge}(9.97 \mathrm{keV}) \mathrm{K}_{\alpha}$ emission lines are detected.
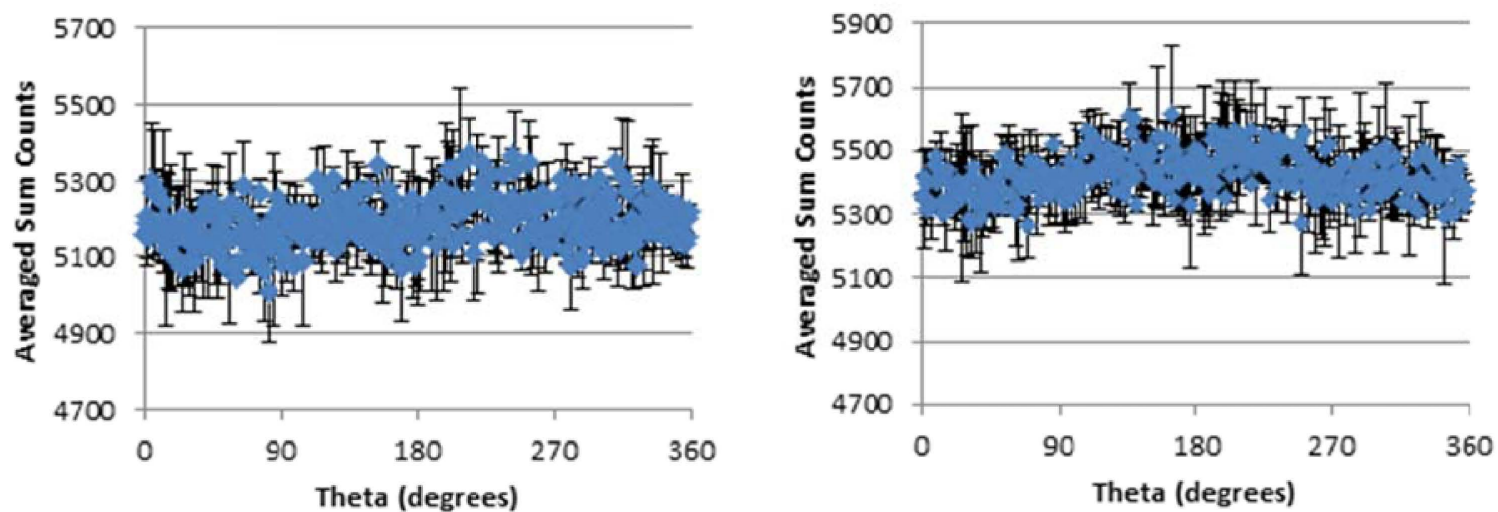

Figure 2. Averaged sum Ga $\mathrm{K}_{\alpha}$ counts (left) and averaged $\mathrm{Ge} \mathrm{K}_{\alpha}$ counts of a DIME capsule as a function of sample rotation. Error bars are standard deviations of the averages.

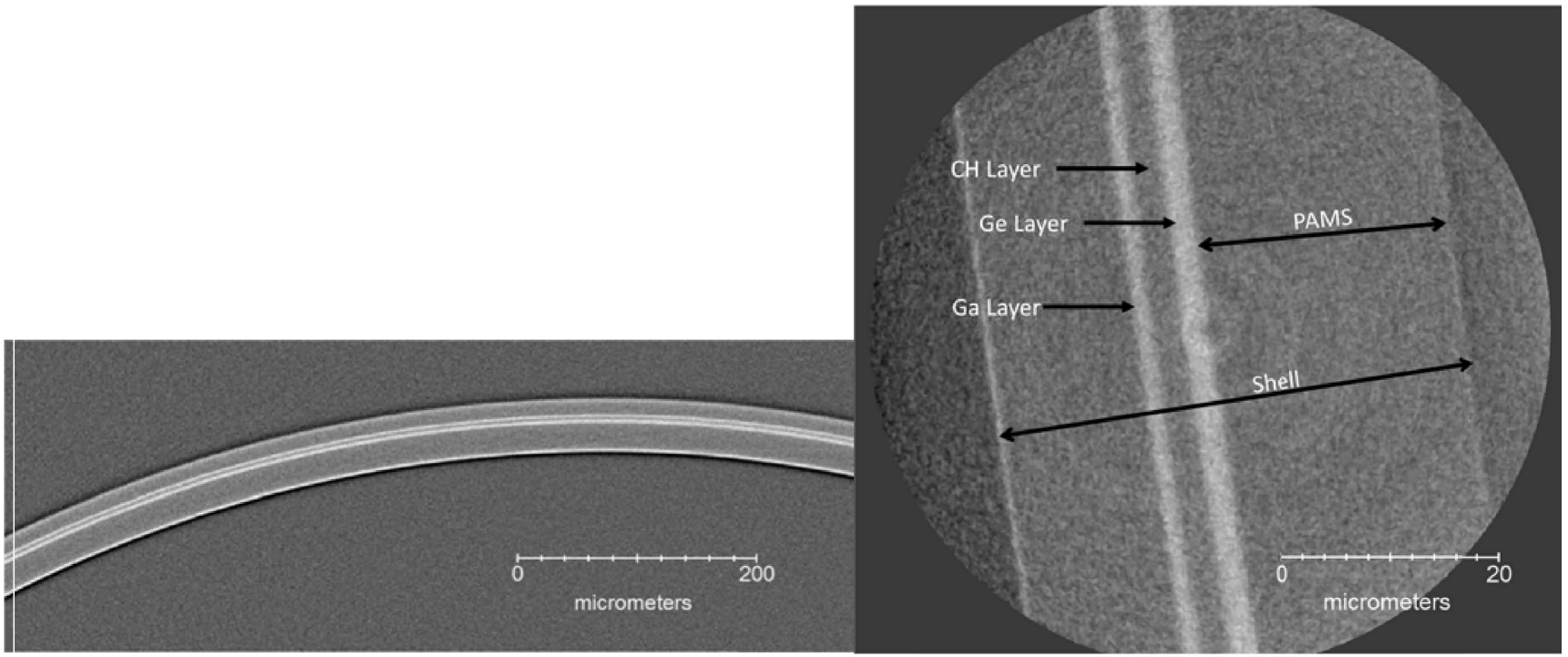

Figure 3. Micro-CT (right, $600 \mathrm{~nm}$ voxel size) and nano-CT (left, $65 \mathrm{~nm}$ voxel size) reconstructed slice images of a DIME capsule. 